INPLASY PROTOCOL

To cite: Wang et al. Effect of Balneology on Patients with Knee Osteoarthritis: A Metaanalysis. Inplasy protocol 2020120140. doi:

10.37766/inplasy2020.12.0140

Received: 29 December 2020

Published: 29 December 2020

Corresponding author:

Lidong Wang

wldwong@outlook.com

Author Affiliation:

Nanjing University of Posts and Telecommunications

Support: None.

Review Stage at time of this submission: Data analysis.

Conflicts of interest: None.

\section{Effect of Balneology on Patients with Knee Osteoarthritis: A Meta-analysis}

Wang, LD'; Xie, X2; Yang, YZh³; Jiang, LP4.

Review question / Objective: Previous systematic reviews of the therapeutic effects of hydrotherapy on KOA patients have not shown positive effects of hydrotherapy on KOA patients due to the limited or multiple inclusion literatures (hip osteoarthritis and knee osteoarthritis). For this reason, it is necessary to update existing studies to confirm the positive effects of hydrotherapy on pain, physical function, and quality of life in KOA patients.

Condition being studied: Osteoarthritis (OA) is the most common musculoskeletal disease, characterized by degenerative cartilage degeneration, and the knee joint is the most common weight-bearing joint. International clinical practice guidelines emphasize the importance of non-drug interventions and recommend education and exercise as primary treatment for hip and knee diseases. As an important method for the treatment of chronic pain, exercise has been shown to have positive changes in patients' symptoms and functional ability, as well as contribute to weight loss, so it is strongly recommended for the treatment of KOA. Previous systematic reviews of the therapeutic effects of hydrotherapy on KOA patients have not shown positive effects of hydrotherapy on KOA patients due to the limited or multiple inclusion literature (hip osteoarthritis and knee osteoarthritis).For this reason, it is necessary to update existing studies to confirm the positive effects of hydrotherapy on pain, physical function, and quality of life in KOA patients.

INPLASY registration number: This protocol was registered with the International Platform of Registered Systematic Review and Meta-Analysis Protocols (INPLASY) on 29 December 2020 and was last updated on 29 December 2020 (registration number INPLASY2020120140).

\section{INTRODUCTION}

Review question / Objective: Previous systematic reviews of the therapeutic effects of hydrotherapy on KOA patients have not shown positive effects of hydrotherapy on KOA patients due to the limited or multiple inclusion literatures (hip osteoarthritis and knee osteoarthritis). For this reason, it is necessary to update 
existing studies to confirm the positive effects of hydrotherapy on pain, physical function, and quality of life in KOA patients.

Condition being studied: Osteoarthritis (OA) is the most common musculoskeletal disease, characterized by degenerative cartilage degeneration, and the knee joint is the most common weight-bearing joint. International clinical practice guidelines emphasize the importance of non-drug interventions and recommend education and exercise as primary treatment for hip and knee diseases. As an important method for the treatment of chronic pain, exercise has been shown to have positive changes in patients' symptoms and functional ability, as well as contribute to weight loss, so it is strongly recommended for the treatment of KOA. Previous systematic reviews of the therapeutic effects of hydrotherapy on KOA patients have not shown positive effects of hydrotherapy on KOA patients due to the limited or multiple inclusion literature (hip osteoarthritis and knee osteoarthritis).For this reason, it is necessary to update existing studies to confirm the positive effects of hydrotherapy on pain, physical function, and quality of life in KOA patients.

\section{METHODS}

Participant or population: knee osteoarthritis (KOA) patients.

Intervention: Studies that compared Balneology with no treatment, usual care, or any active treatment were eligible. No restrictions were made regarding details of Balneology protocol. Co-interventions were allowed.

Comparator: The intervention group was hydrotherapy exercise; the control group had no additional activities, conventional treatment or conservative treatment, and had no specific restrictions on hydrotherapy exercise programs (such as strength and aerobic exercise).

Study designs to be included: Only randomized controlled trials (RCTs) were considered eligible.
Eligibility criteria: Studies with complete data and not republished.

Information sources: PubMed, Cochrane, Embase, Web of Science, CNKI and other databases were searched, and the retrieval date was from July 2020.7.

Main outcome(s): At least one of the pain, stiffness, or physical function of KOA patients should be included, with indicators such as quality of life and physical symptoms extracted as secondary indicators if possible.

Quality assessment / Risk of bias analysis: A data extraction table was prepared, and two searchers independently extracted the basic information of the literature, revalidation information of the eligibility for inclusion in the study, characteristic information of the study object, intervention information, outcome indicators, and outcome information, and evaluated the quality of the study. The data of the last observed value of each study were extracted. In case of disagreement, if the two parties still cannot reach an agreement through negotiation, a third party shall submit the dispute to arbitration for settlement. According to the recommendations of the evidence-based medical research Guidelines, the Cochrane systematic evaluation of "risk assessment for Bias" [15] was used to evaluate literature quality, including random sequence generation, allocation and concealment, blindness, completeness of outcome indicators, selective reporting, and other biases. Look up the study proposal for each trial in the clinical trial registry database and contact the original author if necessary to determine how the trial will be carried out.

Strategy of data synthesis: By using the fixed-effect model and random effect model calculating standardized mean difference (SMD) and $95 \%$ confidence interval $(\mathrm{Cl})$ effect, and through the analysis into the characteristics of literature, using the 12 statistic quantitative evaluation research of heterogeneity, when 12 acuities were $50 \%, P<0.05$, said the study between 
heterogeneity can be accepted, using the fixed-effect model for data analysis; On the contrary, random-effects model is used for data analysis. In this study, the stata15.0 software package was used for statistical analysis.

Subgroup analysis: If necessary, subgroup analysis will be used for heterogeneity test.

Sensibility analysis: Sensitivity analysis by excluding literature one by one.

Language: No language restrictions.

Country(ies) involved: China.

Keywords: Balneology; Osteoarthritis of the knee; Meta-analysis; Review.

Contributions of each author:

Author 1 - Wang Lidong.

Email: vldwong@outlook.com

Author 2 - Xie Xun.

Author 3 - Yang Yizhuo.

Author 4 - Jiang Llping. 